\title{
BMJ Open Reduction in sympathetic tone in patients with obstructive sleep apnoea: is fixed CPAP more effective than APAP? A randomised, parallel trial protocol
}

\author{
Erika Treptow, ${ }^{1}$ Jean Louis Pepin, ${ }^{1,2}$ Sebastien Bailly, ${ }^{1}$ Patrick Levy, ${ }^{1}$ Cecile Bosc ${ }^{3}$ \\ Marie Destors, ${ }^{1,2}$ Holger Woehrle, ${ }^{4}$ Renaud Tamisier ${ }^{1,2}$
}

To cite: Treptow E, Pepin JL, Bailly $\mathrm{S}$, et al. Reduction in sympathetic tone in patients with obstructive sleep apnoea: is fixed CPAP more effective than APAP? A randomised, parallel trial protocol. BMJ Open 2019:9:e024253. doi:10.1136/ bmjopen-2018-024253

- Prepublication history for this paper is available online. To view these files please visit the journal online (http://dx.doi. org/10.1136/bmjopen-2018024253).

Received 18 May 2018 Revised 9 0ctober 2018 Accepted 23 November 2018

Check for updates

(c) Author(s) (or their employer(s)) 2019. Re-use permitted under CC BY-NC. No commercial re-use. See rights and permissions. Published by BMJ.

${ }^{1} \mathrm{HP2}$, Inserm 1042, Université Grenoble Alpes, Grenoble,

France

${ }^{2}$ Laboratoire sommeil, Hôpital Universitaire de Grenoble,

Grenoble, France

${ }^{3}$ Centre Santé et Sommeil,

Grenoble, France

${ }^{4}$ Lung Center Ulm, Ulm, Germany

Correspondence to

Dr Renaud Tamisier;

RTamisier@chu-grenoble.fr

\section{ABSTRACT}

Introduction Obstructive sleep apnoea (OSA) is a prevalent disease associated with cardiovascular events. Hypertension is one of the major intermediary mechanisms leading to long-term cardiovascular adverse events. Intermittent hypoxia and hypercapnia associated with nocturnal respiratory events stimulate chemoreflexes, resulting in sympathetic overactivity and blood pressure (BP) elevation. Continuous positive airway pressure (CPAP) is the primary treatment for OSA and induces a small but significant reduction in BP. The use of autoadjusting positive airway pressure (APAP) has increased in the last years and studies showed different ranges of $\mathrm{BP}$ reduction when comparing both modalities. However, the pathophysiological mechanisms implicated are not fully elucidated. Variations in pressure through the night inherent to APAP may induce persistent respiratory efforts and sleep fragmentation that might impair sympathovagal balance during sleep and result in smaller decreases in BP. Therefore, this double-blind randomised controlled trial aims to compare muscle sympathetic nerve activity (MSNA) assessed by microneurography (reference method for measuring sympathetic activity) after 1 month of APAP versus fixed CPAP in treatment-naive OSA patients. This present manuscript describes the design of our study, no results are presented herein. and is registered under the below reference number.

Methods and analysis Adult subjects with newly diagnosed OSA (Apnoea-Hypopnoea Index >20/hour) will be randomised for treatment with APAP or fixed CPAP. Measurements of sympathetic activity by MSNA, heart rate variability and catecholamines will be obtained at baseline and after 30 days. The primary composite outcome will be the change in sympathetic tone measured by MSNA in bursts/min and bursts/100 heartbeats. Sample size calculation was performed with bilateral assumption. We will use the Student's t-test to compare changes in sympathetic tone between groups.

Ethics and dissemination The protocol was approved by The French Regional Ethics Committee. The study started in March 2018 with primary completion expected to March 2019. Dissemination plans of the results include presentations at conferences and publication in peerreviewed journals.

\section{Strengths and limitations of this study}

- The analysis of vascular and cardiac sympathetic activity will be evaluated by complementary methods: microneurography of the peroneal nerve (the gold-standard method), heart rate variability and urinary catecholamines.

- The use of 24 hours ambulatory blood pressure (BP) monitoring is more sensitive when assessing therapeutic interventions than office BP and provides prognostic guidance.

- A statistician not involved in the data collection will randomly allocate subjects to one of the two positive airway pressure (PAP) modalities. All other investigators, patients and assessment technicians will be blinded to the patient's group.

- The same brand and model of continuous PAP device will be used both in fixed and auto-adjusting PAP modes.

- The duration of exposure to treatment of 1 month is ample but might under-represent the chronic effects of PAP therapies on sympathetic activity.

Trial registration number NCT03428516; Pre-results.

\section{INTRODUCTION}

Obstructive sleep apnoea (OSA) is associated with increased cardiovascular morbidity and mortality. ${ }^{1-6}$ Hypertension is the main intermediary mechanism implicated in cardiovascular risk. ${ }^{7-9}$ There is a dose-response relationship between indices of OSA severity and hypertension. ${ }^{1011}$ Accordingly, one of the most common objectives when treating OSA is blood pressure (BP) reduction in order to prevent or reduce long-term cardiovascular adverse events.

OSA is characterised by partial (hypopnoea) or complete (apnoea) upper airway collapses during sleep. ${ }^{12}$ Sympathetic activation is 
the main intermediary mechanism for BP elevation in patients with OSA. ${ }^{11} 13$ The repetitive occurrence of respiratory events leading to intermittent hypoxia and hypercapnia results in stimulation of central and peripheral chemoreflexes enhancing sympathetic activity and, subsequently increasing vascular tone and promoting BP elevation. ${ }^{14}$ Chronic sympathetic activation induces vascular remodelling and, frequently, uncontrolled or resistant hypertension..$^{15}$ In patients with OSA, night-time sympathetic overactivation is associated with the non-dipping pattern of $\mathrm{BP}$ and the high sympathetic tone persists during wakefulness. ${ }^{16}$ In the early course of the disease, even non-hypertensive subjects exhibit increased BP and muscle sympathetic nerve activity (MSNA) in response to chronic intermittent hypoxia. ${ }^{13} 17$ MSNA is one of the reference methods for measuring sympathetic activity and understanding the pathophysiology of neurogenic hypertension. ${ }^{18}$ Moreover, MSNA changes across time or after intervention are corresponding with arterial BP changes in prehypertension. ${ }^{19}$

In different models of hypertension only intermittent hypoxia, which is the main stimuli in OSA, causes neurogenesis modulation in hippocampus. ${ }^{20}$ In human, intermittent hypoxic exposure induces after 2 and 4 weeks an increase in daytime MSNA. ${ }^{13} 17$ This increase in sympathetic tone was suggested in the early ' 90 s as a mechanism of hypertension in OSA. ${ }^{1621}$ Therefore, MSNA measurement is of particular interest in showing the effect of OSA treatment as a surrogate marker of cardiovascular outcomes. Although several studies have demonstrated the beneficial effects of OSA treatment by continuous positive airway pressure (CPAP) in sympathetic activation, ${ }^{14}$ 22-24 this measurement has never been evaluated in patients under auto-adjusting PAP (APAP). MSNA consists of a technique of microneurography minimally invasive that measures the sympathetic nerve activity of the peroneal nerve.

CPAP remains the gold-standard therapeutic option for the treatment of moderate and severe OSA. Several systematic reviews and meta-analyse ${ }^{25-31}$ have demonstrated limited but significant improvements in BP (a reduction of about $2 \mathrm{~mm} \mathrm{Hg}$ in 24 hours mean BP [MBP]). Better results are achieved in specific phenotypes with more severe OSA, higher BP at baseline and adherent to PAP therapies (use of CPAP $\geq 4$ hours /night). ${ }^{2632-34}$

APAP changes the pressure delivered throughout the night depending on events detected, with the goal of applying the minimal effective pressure ${ }^{35-38}$ thus reducing side effects and improving adherence. The average overnight applied pressure is significantly lower with APAP for the same range of improvement in the Apnoea-Hypopnoea Index (AHI).$^{39}$ However, the continuous variations in pressure associated with the functioning of APAP devices potentially induce microarousals, and change sleep macrostructure in some patients. ${ }^{38} 4041$ This might limit the decrease in sympathetic activity during the night when treating OSA and consequently result in a smaller reduction of $\mathrm{BP}$.
Studies have demonstrated controversial results on the impact of the two pressure modalities (fixed vs auto-adjusting) on BP reduction (table 1). Although some authors reported superiority with fixed pressure,${ }^{4243}$ other clinical trials reported no significant difference between APAP and CPAP. ${ }^{44-46}$ A recent study that evaluated 208 patients with a longer than usual follow-up of 2 years demonstrated comparable reductions in sleepiness and $\mathrm{BP}$ with similar OSA-related costs for both treatments. ${ }^{44}$ Karasulu et at ${ }^{47}$ and Patruno et $a t^{48}$ have demonstrated lower reduction in cardiac sympathetic activity using heart rate variability (HRV) in OSA patients and obese OSA patients during APAP treatment compared with fixed CPAP. However, neither study was randomised and Patruno et al evaluated only a specific population of obese patients with severe OSA, which limits the generalisability of the results. In a small study of adult males, without antihypertensive treatment, Marrone $e t a l^{41}$ evaluated BP changes after treatment with APAP versus CPAP. As a secondary outcome, they reported sympathetic activity by measurement of catecholamines. Norepinephrine decreased significantly after treatment in the APAP group but not in the CPAP group and normetanephrine decreased significantly in both groups. Overall, there is a lack of well-designed studies evaluating the mechanisms underlying specific BP responses under APAP versus fixed CPAP. In this context, the aim of the present study is to compare vascular sympathetic tone after 1 month of treatment with fixed versus auto-adjusting pressure by microneurography in newly diagnosed patients with OSA.

\section{METHODS AND ANALYSIS \\ Study design}

This study is a prospective, single-site, randomised, double-blind, parallel, 1-month controlled trial.

\section{Objectives}

\section{Primary research objective}

The main objective is to compare change in vascular sympathetic tone measured by MSNA microneurography after 1 month of APAP versus after 1 month of fixed CPAP in treatment-naive moderate to severe OSA patients.

The primary composite outcome will be the change in sympathetic tone measured by MSNA in bursts/min and bursts/100 heartbeats between baseline and after 1 month of treatment.

\section{Secondary research objectives}

The secondary objectives will be to compare the following variables before and after treatment:

Ambulatory BP monitoring (24hours ABPM): MBP, systolic BP (SBP) and diastolic BP (DBP), during 24 hours periods as well as daytime and night-time measurements.

Urinary catecholamines (24hours collection): epinephrine, norepinephrine and dopamine.

$\mathrm{HRV}$ as an indicator of cardiac sympathovagal balance. 
Table 1 Literature on the impact of CPAP versus APAP on BP

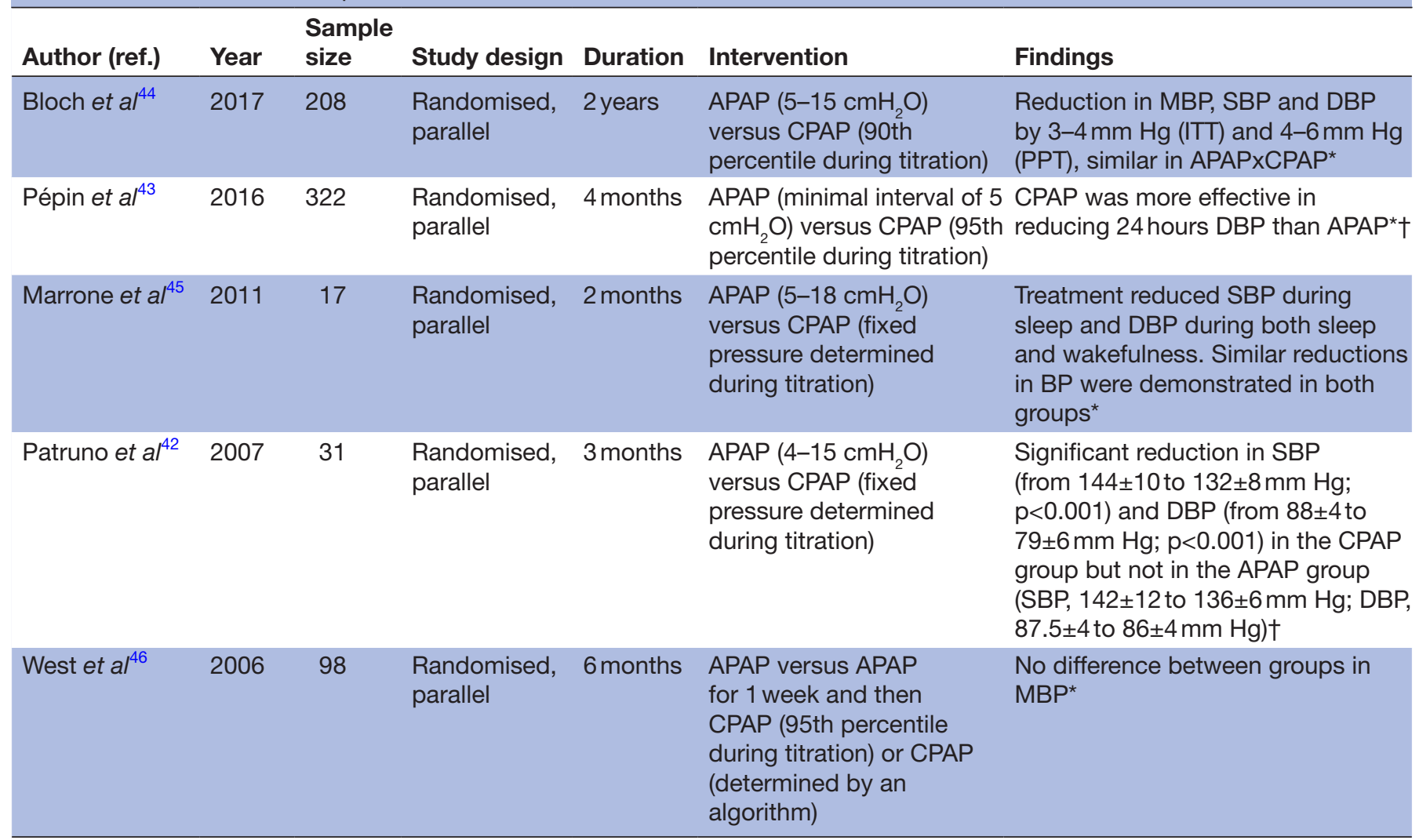

*Ambulatory blood pressure monitoring (24 hours).

†Office blood pressure measurements.

APAP, auto-adjusting positive airway pressure; BP, blood pressure; CPAP, continuous positive pressure; DBP, diastolic blood pressure; ITT, intention to treat; MBP, mean blood pressure; PPT, per-protocol analysis; SBP, systolic blood pressure.

\section{Population}

Consecutive adult outpatients attending a tertiary hospital sleep centre (Grenoble Alpes University Hospital-France), with an established diagnosis of OSA by full-night polysomnography (AHI $>20 /$ hour), daytime sleepiness (Epworth Sleepiness Scale $>10$ ) ${ }^{49}$ and willing to receive PAP treatment, will be invited to participate in the study. The inclusion and exclusion criteria are presented in table 2. Written informed consent will be obtained from all participants by a sleep physician study investigator (see online supplementary file).

\section{Materials}

Muscle sympathetic nerve activity

We will obtain MSNA from nerve recordings using standard tungsten microelectrodes inserted into the peroneal nerve into the popliteal area, after localisation by electric surface stimulation (figure 1). Signals will be filtered (700$2000 \mathrm{~Hz}$ ), amplified (x70 000) and full-wave rectified. The rectified signal will be integrated ( $0.1 \mathrm{~s}$ moving window) for display and for recording (Nerve Traffic Analyzer, Model 662 c-3, University of Iowa, Bioengineering Dept., Iowa City, Iowa, USA). Electrode position in muscle fascicles will be confirmed by pulse synchronous bursts of activity occurring 1.2-1.4s after the ECG QRS complex, reproducible activation during the second phase of the
Valsalva manoeuvre, elicitation of afferent nerve activity by mild muscle stretching and the absence of response to startle. Doppler popliteal vascular flows (DWL500EZ) will be measured during time periods concomitant from measurement of MSNA, HR (three-lead ECG) and arterial BP. Beat-by-beat arterial BP for vascular Doppler

\begin{tabular}{ll}
\hline Table 2 Inclusion and exclusion criteria \\
\hline Inclusion criteria & Exclusion criteria \\
\hline Patients aged 18-80years & Pregnancy \\
\hline OSA (AHI $\geq 20$ events/hour) & $\begin{array}{l}\text { Person deprived of liberty or } \\
\text { subject to a legal protection } \\
\text { measure }\end{array}$ \\
\hline Daytime sleepiness & Patient with heart failure \\
Naive of any pressure & $\begin{array}{l}\text { Patient with central sleep } \\
\text { apnoea index above 20\% of }\end{array}$ \\
Able to provide written & $\begin{array}{l}\text { Patient with unstable } \\
\text { comorbidities that could } \\
\text { influence the results }\end{array}$ \\
\hline
\end{tabular}

Not a vulnerable person or

legally protected adult

AHI, Apnoea-Hypopnoea Index; OSA, obstructive sleep apnoea. 


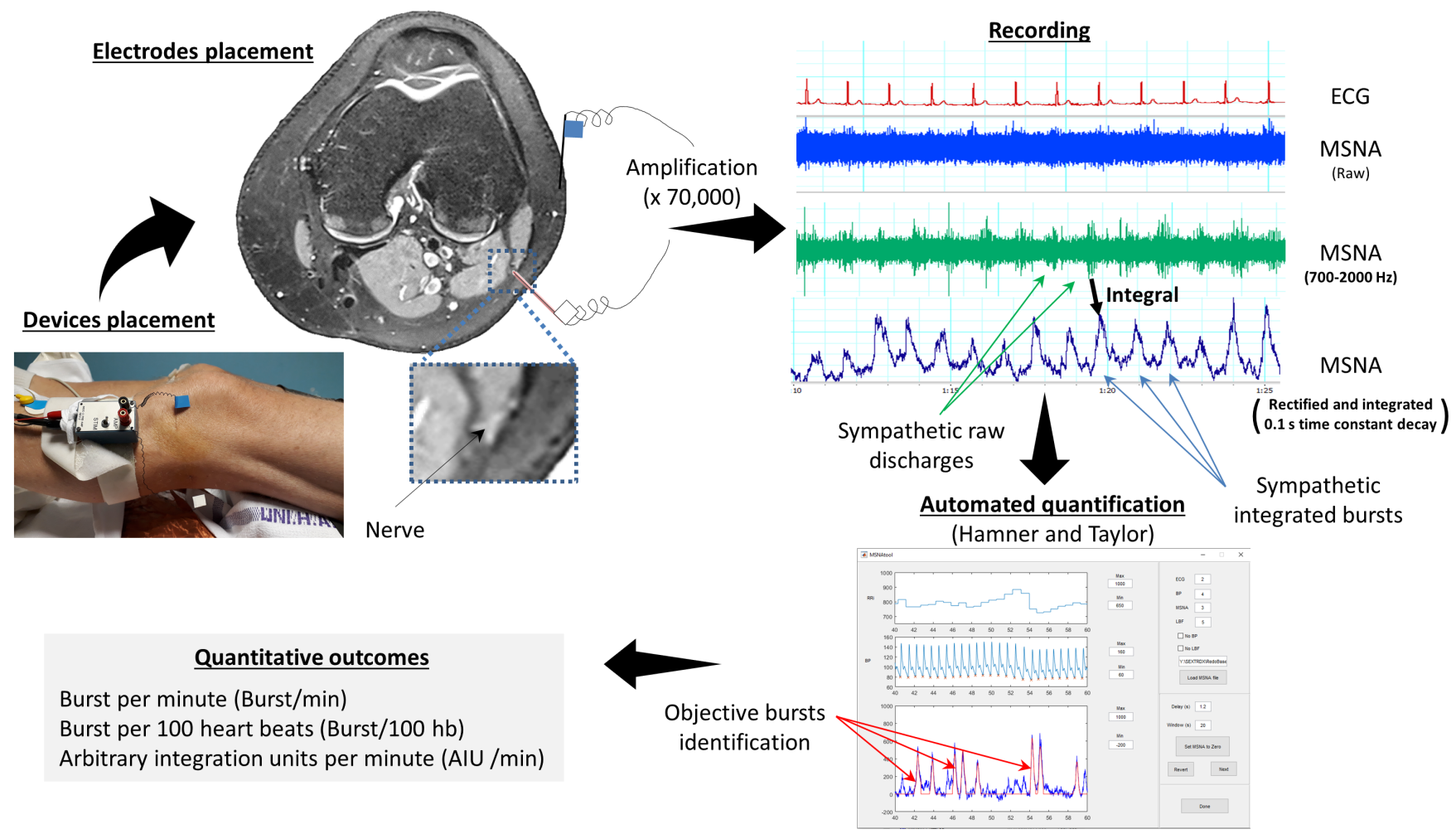

Figure 1 Settings, acquisition, recording and reporting of muscle sympathetic nerve activity (MSNA). Measurement of MSNA is obtained by placement of an uninsulated tungsten register electrode in the peroneal nerve in the popliteal fossae or close to the fibula head. The objective is to reach postganglionic efferent sympathetic neurons. Potential voltage signal is recorded between the nerve electrode and a reference electrode placed on the external side of the knee. The acquired electrical signal is then amplified, band-filtered $(700-2000 \mathrm{~Hz})$, rectified and integrated. Sympathetic bursts, which correspond to nerve firing, are detected and scored using an automatic software in order to minimise subjective interpretation of the signal. MSNA results may be expressed in number of bursts per min or per 100 heartbeats, burst/min and bursts/100 heartbeats, respectively, or using the sum of areas under the curve of all burst in arbitrary integration units per minute or per 100 heartbeats, AUI/min and AUI/100 heartbeats, respectively.

leg resistance calculations will be measured using the CNAP system the same time period. All these signals will be digitalised and recorded for off-line analysis on PowerLab system. These measurements will be performed before and after PAP therapy on morning sessions, fasten since 00:00 hours from foods and any beverage except water, during room air breathing. We will average nerve activity, heart rate and arterial BP over 5 min windows of data collection at baseline and post-PAP therapy. Sympathetic bursts will be identified using a specific algorithm described by Hamner and Taylor ${ }^{50}$ using Matlab software (The Mathworks, Natick, Massachusetts, USA). For purposes of quantification, MSNA will be reported in at least 5 min periods and expressed as burst frequency (bursts/min and bursts/100 heartbeats).

\section{Blood flow}

Popliteal blood velocity will be recorded using a $4 \mathrm{MHz}$ Doppler probe (Multidop T2, DWL) at the popliteal fossa of the contralateral leg to the sympathetic nerve recording.

Several parameters will be extracted from the above measurements: sympathetic vascular tone, BP and blood flow. Furthermore, we shall calculate vascular resistance and vascular sympathetic tone and BP gains, as previously described. ${ }^{1451}$

\section{Blood pressure}

ABPM will be measured in the dominant arm over 24 hours at $15 \mathrm{~min}$ intervals during daytime and every $30 \mathrm{~min}$ night-time (ABP monitor 90207, Spacelabs Healthcare, Issaquah, Washington, USA). BP acquisition will begin at a morning session and will finish 24 hours later. The following ABPM parameters will be studied: mean SBP, mean DBP and mean HR over 24 hours, the same mean values during the daytime (07:00 to 22:00 hours) and at night-time (22:00 to 7:00 hours). The normal night-time physiological dipping BP is expected to be $>10 \%$. The summary values in the ABPM report for each patient will be used in the data analysis. This is an average by subject and by recording session (at baseline and 1 month). Data relating to the average daytime and night-time SBP, diastolic $\mathrm{BP}$ (DBP) and MBP will be recorded. SBP values of $>260 \mathrm{~mm} \mathrm{Hg}$ or $<70 \mathrm{~mm} \mathrm{Hg}$ and DBP $>150 \mathrm{~mm} \mathrm{Hg}$ or $<40 \mathrm{~mm} \mathrm{Hg}$ will be automatically eliminated. Daytime hypertension is defined as daytime $\mathrm{SBP}>135 \mathrm{~mm} \mathrm{Hg}$ and/or DBP $>85 \mathrm{~mm} \mathrm{Hg}$, and night-time hypertension as $\mathrm{SBP}>120 \mathrm{~mm} \mathrm{Hg}$ and/or DBP $>70 \mathrm{~mm} \mathrm{Hg}{ }^{52}$ 
Clinical BPs (SBP and DBP) will be measured by mercury sphygmomanometer on three occasions in line with the European Society of Hypertension-European Society of Cardiology and American College of Cardiology/American Heart Association guidelines. ${ }^{9}{ }^{53}$ Mean arterial $\mathrm{BP}$ will be calculated as DBP $+1 / 3$ (SBP-DBP) .

Each recording will be validated only if the following quality criteria are met: cuff size adapted to the diameter of the arm, calibration of the device, full 24 hours duration of recording comprising at least 48 valid measures and no more than two missing time slots.

\section{Catecholamine measurements}

Twenty-four hours urine samples will be collected, acidified with acetic acid and stored at $-20^{\circ} \mathrm{C}$ until analysis. Catecholamines (epinephrine, norepinephrine and dopamine) will be measured in $1 \mathrm{~mL}$ of urine by high-performance liquid chromatography with electrochemical detection (Coularray Detector, ESA Dionex, Chelmsford, USA).

\section{Polysomnography}

Full-night polysomnography will be performed at our sleep laboratory. The following physiological variables will be monitored: electroencephalography (EEG), electrooculography, electromyography, ECG, oral and nasal airflows, chest and abdominal respiratory effort through inductance plethysmography, snoring, body position, oxyhaemoglobin saturation by pulse oximetry and heart rate. Continuous recordings will be taken with electrode positions C3/A2-C4/A1-Cz/01 of the international 10-20 Electrode Placement System, along with eye movements, chin electromyogram and ECG with a modified V2 lead. Airflow will be measured with nasal pressure prongs together with the sum of oral and nasal thermistor signals. Respiratory effort will be monitored using abdominal and thoracic bands. Oxygen saturation will be measured using a pulse oximeter. Respiratory events will be classified according to the American Academy of Sleep Medicine's guidelines. ${ }^{54}$ An apnoea is defined as the complete cessation of airflow for at least $10 \mathrm{~s}$ and hypopnoea as a reduction of at least $30 \%$ in the nasal pressure signal associated with either oxygen desaturation of $\geq 3 \%$ or an EEG arousal from sleep, both lasting for at least $10 \mathrm{~s}$. Apnoeas will be classified as obstructive, central or mixed according to the presence or absence of respiratory effort. The classification of hypopnoeas as obstructive or central will be based on the thoracoabdominal band signal and the shape of the nasal respiratory pressure curve (flow limited aspect or not). The AHI is defined as the number of apnoeas and hypopnoeas per hour of sleep. Sleep will be scored manually according to American Accademy of Sleep Medicine (AASM) criteria. $^{54}$

\section{Procedures}

At the baseline visit, MSNA, ABPM, calf blood flow, HRV and catecholamines will be measured. Then, during a 1-week titration phase, optimal CPAP pressure will be obtained over eight nights at home using an auto CPAP device (RESMED) to obtain a fixed CPAP pressure value. The optimal pressure (95th percentile) will be determined by one expert researcher, based on visual evaluation of the raw data recordings from nights with no significant leaks. The pressure determined during the titration nights will be used as the therapeutic pressure in the fixed CPAP mode, whereas in APAP the pressure level will be adjusted between a minimal pressure of 6 $\mathrm{cmH}_{2} \mathrm{O}$ and a maximum of $16 \mathrm{cmH}_{2} \mathrm{O}$. After the titration phase, participants with a minimum usage of 4 hours per night will be randomised to treatment with either APAP or fixed CPAP. During treatment, the healthcare provider of the device (Agiradom) will be responsible for solving potential problems with usage (eg, mask leaks, side effects). The healthcare provider employees are trained in research good clinical practice, and how to maintain the blindness of allocation to patients and researchers during randomised trials. Finally, following 1 month of treatment, MSNA, ABPM, calf blood flow, HRV and catecholamines will be measured for comparison with baseline. Figure 2 shows the study schema.

\section{Statistical considerations}

\section{Sample size}

We powered the study based on the MSNA outcome. To date, no previous study has compared these two PAP modalities using MSNA as primary outcome. Since there are no reliable MSNA data available, we hypothesised the impact of CPAP to be $8 \pm 5$ bursts/min in one arm and $5 \pm 5$ bursts $/ \mathrm{min}$ in the other arm with no a priori assumption. Assuming an alpha error of $5 \%$, a statistical power of $80 \%$, in bilateral situation, 34 patients per arm will need to be enrolled in the study. In the sample size, we anticipate that $10 \%$ will not meet the criteria of compliance to pressure support after 1 week, and 10\% more will drop out before termination of the study.

Because it is a pilot study, we will perform a group sequential design, first an interim analysis will be performed on data from the first 24 patients (12 per arm) with a nominal $p$ value of 0.0081 required to demonstrate a significant difference between groups. If at the interim analysis, the observed $p$ value for the primary outcome is greater than the nominal $p$ value inclusions will continue until the final sample of 68 patients is reached. Conversely, if significance is observed, the patient inclusion will stop and no further inclusions will be needed. This interim analysis will be performed by a blinded statistician which have no regard on the randomisation list neither contacts with the investigator nor involvement in the study.

\section{Randomisation}

After titration of fixed CPAP (lasting eight nights), participants with a minimum usage of 4 hours per night will be randomised to either fixed CPAP or APAP treatment. Randomisation will be conducted by a statistician independent of the study using a computer-generated random numbers list (six patients per block). Randomisation 


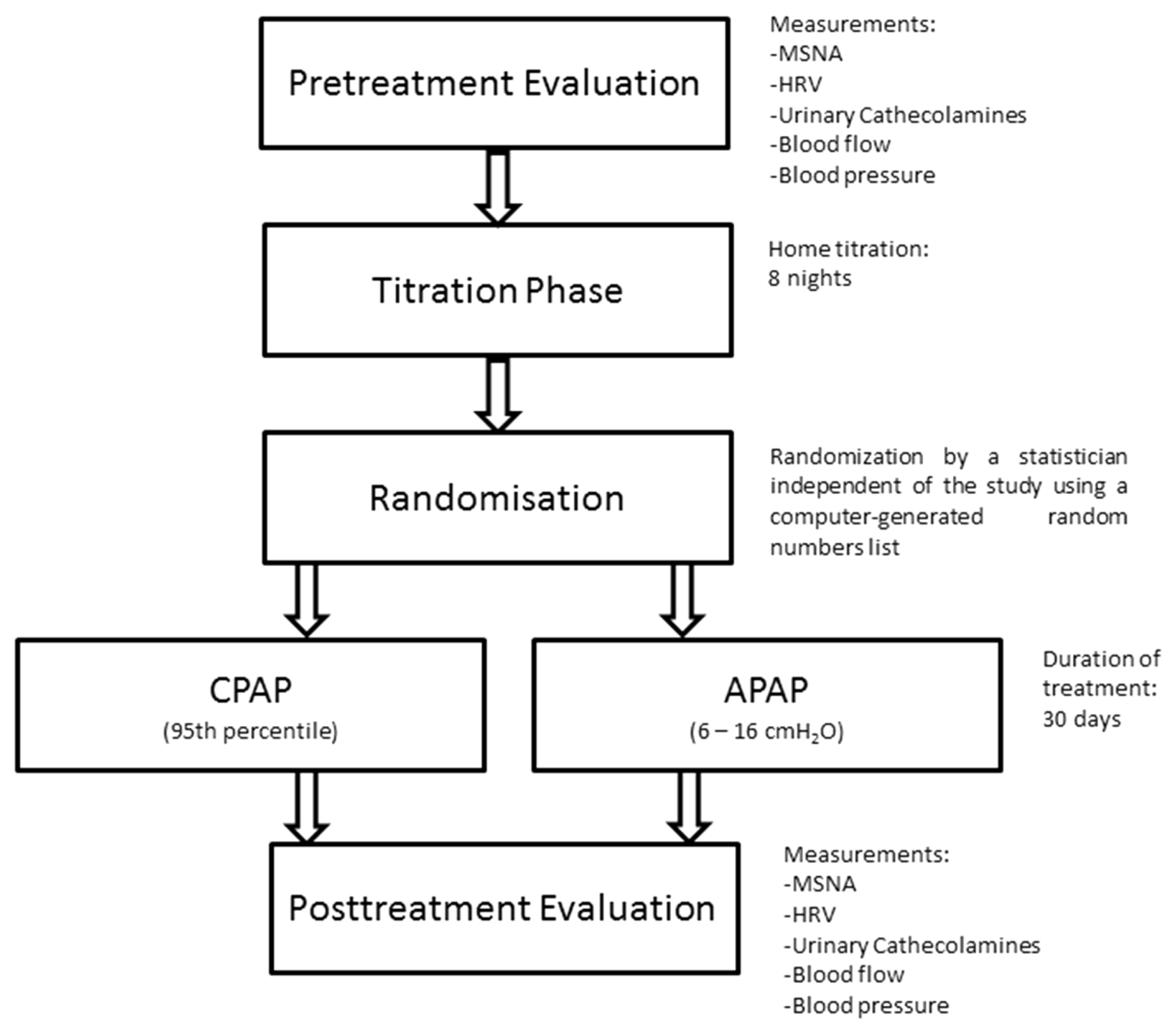

Figure 2 Study protocol. APAP, auto-adjusting positive airway pressure; CPAP, continuous positive airway pressure; HRV, heart rate variability; MSNA, muscle sympathetic nerve activity.

list was provided by the clinical research department of Grenoble Alpes university hospital and the randomisation list is held and followed by two independent persons from the study. These persons provide allocation directly to the healthcare provider maintaining blindness of the patient and the investigators.

\section{Statistical methodology and analyses}

The analysis will be done following the Intention to treat method. Continuous variables will be expressed as median (25th/75th percentiles) or mean (SD), while categorical variables will be reported as absolute numbers and percentages for both groups. Baseline comparisons between groups will be made using a Student's t-test or Mann-Whitney U test, depending on validation of normal distribution. For discrete variables, a $\chi^{2}$ test will be used. Normality will be assessed using the Shapiro-Wilk test. If significant differences are observed between arms, a multivariable regression will be performed. In the case of missing data, an imputation strategy will be applied according to the percentage of missing values. If less than $5 \%$ of missing value are observed, simple imputation will be performed, based on the median for quantitative variables or on the most frequent values for qualitative variables. If the proportion of missing values is between $5 \%$ and $20 \%$, multiple imputations will be performed by using Markov Chain Monte Carlo (MCM) chains for qualitative variables or full conditional specification for quantitative variables. Variables with more than $20 \%$ of missing values will not be taken into account. The main outcome, difference between 1 month and baseline values will be analysed by using a Student's t-test. In the case of multivariable analysis, a linear mixed model will be performed by including a random effect for the patient.

Data management and statistical analyses will be performed using SAS (V.9.4, SAS Institute).

\section{Ethics}

The current study will be conducted in accordance with the Declaration of Helsinki and the recommendations for Good Clinical Practice. Written informed consent (see online supplemental file) will be signed by all study participants before enrolment in the study. Patients have the right to withdraw from the study without incurring any prejudice at any time. The study started in March 2018 with primary completion expected in March 2019.

\section{Patient and public involvement}

Patients, collaborators and sponsors were not directly involved in the design, recruitment and conduction of the study. Dissemination plans of the results include presentations at conferences and publication in peer-reviewed 
journals. Updates of the randomised trial will be available at ClinicalTrials.com. All patients will be informed that the dissemination of results will be accessible on request.

\section{Sponsor and funding}

The sponsor of the study is Grenoble Alpes University Hospital, France. The principal investigator is Renaud Tamisier. Erika Treptow is supported by the Coordenação de Aperfeiçoamento de Pessoal de Nível Superior (CAPES) - Brazil. ResMed, 'Agir pour les maladies chroniques' and the French National Research Agency in the framework of the 'Investissements d'avenir' program (ANR-15-IDEX-02) will provide unrestricted funding. The collaborators and sponsors were not involved in the design of the study and will not influence the execution, analysis and publication of results.

\section{DISCUSSION}

CPAP remains the first-line therapy for patients with OSA. In order to determine the optimal pressure that will maintain airway patency during sleep, a titration made during full-night polysomnography is required. However, this is costly, demands technical expertise and may result in a delay in the initiation of treatment. Furthermore, night-time AHI can be variable, depending on position, sleep architecture, overnight rostral fluid shifts and alcohol intake, which may influence the determination of the optimal effective pressure if performed during only one night. ${ }^{556}$ Therefore, over the last few years, there has been an exponential increase of the use of APAP for automatic titration prior to long-term treatment at home. This simplified procedure is associated with comparable outcomes $^{57}$ and a significant cost reduction. ${ }^{58}$

Currently, in many countries especially in Europe, APAP is by far the most commonly used device for OSA treatment and gives a slight increase in PAP adherence. ${ }^{59}$ However, the increase in APAP use is accompanied by unresolved scientific questions: is APAP as effective as fixed CPAP in reducing cardiovascular risk? Is it safe to use APAP in patients with comorbidities? Do APAP and CPAP have the same efficacy in normalising sleep in patients with OSA? The proposed study will focus on the cardiovascular response to these different PAP modalities. To achieve this, we shall use complementary reference tools to evaluate sympathetic activity in patients using fixed CPAP or APAP. Vascular and cardiac sympathetic activity will be explored in addition to circulating catecholamine levels.

Since its first description in $1967,{ }^{60}$ vascular sympathetic activity measured by microneurography has provided insights into our understanding of the pathophysiology of hypertension, cardiac failure and sleep apnoea. ${ }^{16} 1861$ This method allows the recording of impulses in peripheral nerves and is the gold standard for measurement of vascular sympathetic activity. When applied by experienced professionals, MSNA is reproducible and allows evaluation between subjects before and after an intervention, with minor risks and side effects. ${ }^{51}{ }^{62}$ It has been demonstrated that intermittent hypoxia is a major contributor to inducing sympathetic activation in healthy humans and patients with OSA ${ }^{131763}$ and that treatment with CPAP lowers MSNA. ${ }^{23}$ One of the strengths of the present study is that, in addition to MSNA, we will measure HRV and urinary catecholamines to assess cardiac and whole body sympathetic activity, respectively.

Moreover, we will provide a better understanding of $\mathrm{BP}$ responses under the two PAP therapies and possibly identify the type of patients who would benefit the most from APAP.

The sympathetic APAP-CPAP protocol is a key randomised controlled trial that will assess, for the first time, different PAP modalities that might differ in terms of the decrease in sympathetic activity they induce in patients with OSA. The results of the APAP-CPAP study should provide further clarification as to the cardiovascular benefits of an effective treatment for patients with OSA. In addition, the findings might have important implications for individualised therapeutic strategies by identifying the best phenotypes to be treated by a given PAP therapy.

Acknowledgements The authors would like to thank Marie Peeters for trial management and Alison Foote (Grenoble Alpes University Hospital, Research division) for language revision.

Contributors ET participated in the design of the study, wrote the article based on the study protocol, will include patients, collect and analyse data into the protocol. JLP and PL designed the study, wrote the study protocol, critically revised the manuscript and will include patients into the protocol. SB participated in the design of the protocol, established the statistical analysis plan and calculated the sample size. CB revised the manuscript and will include patients into the protocol. MD revised the manuscript and will include patients into the protocol. HW participated in the design of the study and critically revised the manuscript. RT designed the study, wrote the study protocol and article, critically revised the manuscript and will include patients, and collect and analyse data. The submitted manuscript has been approved by all authors.

Funding ET is supported by Coordenação de Aperfeiçoamento de Pessoal de Nível Superior (CAPES) - Brazil. This work was supported by unrestricted grant from ResMed, by the foundation Agir pour les maladies chroniques and by the French National Research Agency in the framework of the 'Investissements d'avenir' program (ANR-15-IDEX-02).

Competing interests RT reports travel grants from Agiradom (a Home Healthcare provider) and research grants from Resmed.

Patient consent for publication Obtained.

Ethics approval The protocol was approved by The French Regional Ethics Committee (Comite de Protection des Personnes Sud Est V No IRB: 0006705 on 19 February 2018).

Provenance and peer review Not commissioned; externally peer reviewed.

Open access This is an open access article distributed in accordance with the Creative Commons Attribution Non Commercial (CC BY-NC 4.0) license, which permits others to distribute, remix, adapt, build upon this work non-commercially, and license their derivative works on different terms, provided the original work is properly cited, appropriate credit is given, any changes made indicated, and the use is non-commercial. See: http://creativecommons.org/licenses/by-nc/4.0/.

\section{REFERENCES}

1. Baguet JP, Barone-Rochette G, Tamisier R, et al. Mechanisms of cardiac dysfunction in obstructive sleep apnea. Nat Rev Cardiol 2012;9:679-88. 
2. Yaggi HK, Concato J, Kernan WN, et al. Obstructive sleep apnea as a risk factor for stroke and death. N Engl J Med 2005;353:2034-41.

3. Gami AS, Olson EJ, Shen WK, et al. Obstructive sleep apnea and the risk of sudden cardiac death: a longitudinal study of 10,701 adults. $J$ Am Coll Cardiol 2013;62:610-16.

4. Sundström J, Neal B. Effects of Blood Pressure Reduction in Mild Hypertension. Ann Intern Med 2015;163:67-8.

5. Drager LF, McEvoy RD, Barbe F, et al. Sleep Apnea and Cardiovascular Disease: Lessons From Recent Trials and Need for Team Science. Circulation 2017;136:1840-50.

6. Javaheri S, Barbe F, Campos-Rodriguez F, et al. Sleep Apnea: Types, Mechanisms, and Clinical Cardiovascular Consequences. J Am Coll Cardiol 2017;69:841-58.

7. Lim SS, Vos T, Flaxman AD, et al. A comparative risk assessment of burden of disease and injury attributable to 67 risk factors and risk factor clusters in 21 regions, 1990-2010: a systematic analysis for the Global Burden of Disease Study 2010. Lancet 2012;380:2224-60.

8. Danaei G, Finucane MM, Lin JK, et al. National, regional, and global trends in systolic blood pressure since 1980: systematic analysis of health examination surveys and epidemiological studies with 786 country-years and 5.4 million participants. Lancet 2011;377:568-77.

9. Whelton PK, Carey RM, Aronow WS, et al. 2017 ACC/AHA/AAPA ABC/ACPM/AGS/APhA/ASH/ASPC/NMA/PCNA Guideline for the Prevention, Detection, Evaluation, and Management of High Blood Pressure in Adults: Executive Summary: A Report of the American College of Cardiology/American Heart Association Task Force on Clinical Practice Guidelines. Hypertension 2018;71:1269-324.

10. Peppard PE, Young T, Palta M, et al. Prospective study of the association between sleep-disordered breathing and hypertension. $N$ Engl J Med 2000;342:1378-84.

11. Narkiewicz K, Somers VK. Obstructive sleep apnea as a cause of neurogenic hypertension. Curr Hypertens Rep 1999;1:268-73.

12. Lévy P, Kohler M, McNicholas WT, et al. Obstructive sleep apnoea syndrome. Nat Rev Dis Primers 2015;1:15015.

13. Tamisier R, Pépin JL, Rémy J, et al. 14 nights of intermittent hypoxia elevate daytime blood pressure and sympathetic activity in healthy humans. Eur Respir J 2011;37:119-28.

14. Tamisier R, Tan CO, Pepin JL, et al. Blood Pressure Increases in OSA due to Maintained Neurovascular Sympathetic Transduction: Impact of CPAP. Sleep 2015;38:1973-80.

15. Tamisier R, Lévy P, Pépin JL. Do patients with obstructive sleep apnoea deserve new dedicated antihypertensive strategies? Thorax 2017;72:495-7.

16. Somers VK, Dyken ME, Clary MP, et al. Sympathetic neural mechanisms in obstructive sleep apnea. J Clin Invest 1995;96:1897-904.

17. Gilmartin GS, Lynch M, Tamisier R, et al. Chronic intermittent hypoxia in humans during 28 nights results in blood pressure elevation and increased muscle sympathetic nerve activity. Am J Physiol Heart Circ Physiol 2010;299:H925-31.

18. Shoemaker JK, Klassen SA, Badrov MB, et al. Fifty years of microneurography: learning the language of the peripheral sympathetic nervous system in humans. J Neurophysiol 2018;119:1731-44.

19. Hering D, Kucharska W, Chrostowska M, et al. Age-dependent sympathetic neural responses to $B$, selective beta-blockade in untreated hypertension-related tachycardia. Blood Press 2018;27:158-65

20. Pedroso D, Nunes AR, Diogo LN, et al. Hippocampal neurogenesis response: What can we expect from two different models of hypertension? Brain Res 2016;1646:199-206.

21. Carlson JT, Hedner J, Elam M, et al. Augmented resting sympathetic activity in awake patients with obstructive sleep apnea. Chest 1993:103:1763-8.

22. Usui K, Bradley TD, Spaak J, et al. Inhibition of awake sympathetic nerve activity of heart failure patients with obstructive sleep apnea by nocturnal continuous positive airway pressure. J Am Coll Cardiol 2005;45:2008-11.

23. Narkiewicz K, Kato M, Phillips BG, et al. Nocturnal continuous positive airway pressure decreases daytime sympathetic traffic in obstructive sleep apnea. Circulation 1999;100:2332-5.

24. Waradekar NV, Sinoway LI, Zwillich CW, et al. Influence of treatment on muscle sympathetic nerve activity in sleep apnea. Am J Respir Crit Care Med 1996;153(4 Pt 1):1333-8.

25. Bratton DJ, Gaisl T, Wons AM, et al. CPAP vs Mandibular Advancement Devices and Blood Pressure in Patients With Obstructive Sleep Apnea: A Systematic Review and Meta-analysis. JAMA 2015;314:2280-93.

26. Bratton DJ, Stradling JR, Barbé F, et al. Effect of CPAP on blood pressure in patients with minimally symptomatic obstructive sleep apnoea: a meta-analysis using individual patient data from four randomised controlled trials. Thorax 2014;69:1128-35.

27. Montesi SB, Edwards BA, Malhotra A, et al. The effect of continuous positive airway pressure treatment on blood pressure: a systematic review and meta-analysis of randomized controlled trials. J Clin Sleep Med 2012;8:587-96.

28. Alajmi M, Mulgrew AT, Fox J, et al. Impact of continuous positive airway pressure therapy on blood pressure in patients with obstructive sleep apnea hypopnea: a meta-analysis of randomized controlled trials. Lung 2007;185:67-72.

29. Bazzano LA, Khan Z, Reynolds K, et al. Effect of nocturnal nasal continuous positive airway pressure on blood pressure in obstructive sleep apnea. Hypertension 2007;50:417-23.

30. Haentjens P, Van Meerhaeghe A, Moscariello A, et al. The impact of continuous positive airway pressure on blood pressure in patients with obstructive sleep apnea syndrome: evidence from a metaanalysis of placebo-controlled randomized trials. Arch Intern Med 2007;167:757-64.

31. McDaid C, Durée KH, Griffin SC, et al. A systematic review of continuous positive airway pressure for obstructive sleep apnoeahypopnoea syndrome. Sleep Med Rev 2009;13:427-36.

32. Martínez-García MA, Capote F, Campos-Rodríguez F, et al. Effect of CPAP on blood pressure in patients with obstructive sleep apnea and resistant hypertension: the HIPARCO randomized clinical trial. JAMA 2013;310:2407-15.

33. Barbé F, Durán-Cantolla J, Sánchez-de-la-Torre M, et al. Effect of continuous positive airway pressure on the incidence of hypertension and cardiovascular events in nonsleepy patients with obstructive sleep apnea: a randomized controlled trial. JAMA 2012;307:2161-8.

34. Pépin JL, Timsit JF, Tamisier R, et al. Is CPAP effective in reducing blood pressure in minimally symptomatic obstructive sleep apnoea? Thorax 2014;69:1068-70.

35. Sanders $\mathrm{MH}$, Montserrat JM, Farré R, et al. Positive pressure therapy: a perspective on evidence-based outcomes and methods of application. Proc Am Thorac Soc 2008;5:161-72.

36. Hertegonne K, Bauters F. The value of auto-adjustable CPAP devices in pressure titration and treatment of patients with obstructive sleep apnea syndrome. Sleep Med Rev 2010;14:115-9.

37. Morgenthaler TI, Aurora RN, Brown T, et al. Practice parameters for the use of autotitrating continuous positive airway pressure devices for titrating pressures and treating adult patients with obstructive sleep apnea syndrome: an update for 2007. An American Academy of Sleep Medicine report. Sleep 2008;31:141-7.

38. Won CH. Man Versus Machine. J Clin Sleep Med 2017;13:167-8.

39. Ayas NT, Patel SR, Malhotra A, et al. Auto-titrating versus standard continuous positive airway pressure for the treatment of obstructive sleep apnea: results of a meta-analysis. Sleep 2004;27:249-53.

40. Fuchs FS, Wiest GH, Frank M, et al. Auto-CPAP therapy for obstructive sleep apnea: induction of microarousals by automatic variations of CPAP pressure? Sleep 2002;25:514-8.

41. Marrone O, Insalaco G, Bonsignore MR, et al. Sleep structure correlates of continuous positive airway pressure variations during application of an autotitrating continuous positive airway pressure machine in patients with obstructive sleep apnea syndrome. Chest 2002;121:759-67.

42. Patruno V, Aiolfi S, Costantino G, et al. Fixed and autoadjusting continuous positive airway pressure treatments are not similar in reducing cardiovascular risk factors in patients with obstructive sleep apnea. Chest 2007;131:1393-9.

43. Pépin JL, Tamisier R, Baguet JP, et al. Fixed-pressure CPAP versus auto-adjusting CPAP: comparison of efficacy on blood pressure in obstructive sleep apnoea, a randomised clinical trial. Thorax 2016;71:726-33.

44. Bloch KE, Huber F, Furian M, et al. Autoadjusted versus fixed CPAP for obstructive sleep apnoea: a multicentre, randomised equivalence trial. Thorax 2018;73.

45. Marrone $\mathrm{O}$, Salvaggio A, Bue AL, et al. Blood pressure changes after automatic and fixed CPAP in obstructive sleep apnea: relationship with nocturnal sympathetic activity. Clin Exp Hypertens 2011;33:373-80.

46. West SD, Jones DR, Stradling JR. Comparison of three ways to determine and deliver pressure during nasal CPAP therapy for obstructive sleep apnoea. Thorax 2006;61:226-31.

47. Karasulu L, Epöztürk PO, Sökücü SN, et al. Improving Heart rate variability in sleep apnea patients: differences in treatment with autotitrating positive airway pressure (APAP) versus conventional CPAP. Lung 2010;188:315-20.

48. Patruno V, Tobaldini E, Bianchi AM, et al. Acute effects of autoadjusting and fixed continuous positive airway pressure treatments on cardiorespiratory coupling in obese patients with obstructive sleep apnea. Eur J Intern Med 2014;25:164-8. 
49. Johns MW. A new method for measuring daytime sleepiness: the Epworth sleepiness scale. Sleep 1991;14:540-5.

50. Hamner JW, Taylor JA. Automated quantification of sympathetic beat-by-beat activity, independent of signal quality. J Appl Physiol 2001;91:1199-206.

51. Tan CO, Tamisier R, Hamner JW, et al. Characterizing sympathetic neurovascular transduction in humans. PLoS One 2013;8:e53769.

52. Williams B, Mancia G, Spiering W, et al. 2018 ESC/ESH Guidelines for the management of arterial hypertension: The Task Force for the management of arterial hypertension of the European Society of Cardiology and the European Society of Hypertension: The Task Force for the management of arterial hypertension of the European Society of Cardiology and the European Society of Hypertension. $J$ Hypertens 2018;36:1953-2041.

53. Mancia G, De Backer G, Dominiczak A et al. 2007 Guidelines for the Management of Arterial Hypertension: The Task Force for the Management of Arterial Hypertension of the European Society of Hypertension (ESH) and of the European Society of Cardiology (ESC). J Hypertens 2007;25:1105-87.

54. Berry RB, Brooks R, Gamaldo C, et al. AASM Scoring Manual Updates for 2017 (Version 2.4). J Clin Sleep Med 2017;13:665-6.

55. White LH, Lyons OD, Yadollahi A, et al. Night-to-night variability in obstructive sleep apnea severity: relationship to overnight rostral fluid shift. J Clin Sleep Med 2015;11:149-56.

56. Bittencourt LR, Suchecki D, Tufik $S$, et al. The variability of the apnoea-hypopnoea index. J Sleep Res 2001;10:245-51.
57. Stradling JR, Barbour C, Pitson DJ, et al. Automatic nasal continuous positive airway pressure titration in the laboratory: patient outcomes. Thorax 1997;52:72-5.

58. Corral J, Sánchez-Quiroga MÁ, Carmona-Bernal C, et al. Conventional Polysomnography Is Not Necessary for the Management of Most Patients with Suspected Obstructive Sleep Apnea. Noninferiority, Randomized Controlled Trial. Am J Respir Crit Care Med 2017;196:1181-90.

59. Smith I, Nadig V, Lasserson TJ. Educational, supportive and behavioural interventions to improve usage of continuous positive airway pressure machines for adults with obstructive sleep apnoea. Cochrane Database Syst Rev 2009;2:CD007736.

60. Vallbo AB, Hagbarth KE. Impulses recorded with micro-electrodes in human muscle nerves during stimulation of mechanoreceptors and voluntary contractions. Electroencephalogr Clin Neurophysiol 1967;23:392.

61. Mark AL, Wallin BG. Microneurography: a technique for assessing central neural effects of adrenergic drugs on sympathetic outflow in humans. J Cardiovasc Pharmacol 1985;7 Suppl 8(Suppl 8):S67-9.

62. Kimmerly DS, O'Leary DD, Shoemaker JK. Test-retest repeatability of muscle sympathetic nerve activity: influence of data analysis and head-up tilt. Auton Neurosci 2004;114(1-2):61-71.

63. Xie A, Skatrud JB, Crabtree DC, et al. Neurocirculatory consequences of intermittent asphyxia in humans. $J$ Appl Physiol 2000;89:1333-9. 